\title{
Quantum Information Transmission Using CNOT Gate
}

\author{
Ankit Sharma ${ }^{\mathrm{a}, 1}$ and Manisha J Nene ${ }^{\mathrm{a}}$ \\ ${ }^{a}$ Department of Computer Science \& Engineering Defense Institute of Advanced \\ Technology, Pune, Maharashtra, India
}

\begin{abstract}
We are at the dawn of quantum era; research efforts are been made on quantum information transmission techniques. Properties of quantum mechanics poses unique challenges in terms of wave collapse function, No cloning theorem and reversible operations. Quantum teleportation and quantum entanglement swapping based architecture are utilized to transmit qubit. In this paper we propose an approach to transmit qubits using controlled NOT gate (CNOT) gates and implement it on quantum machine.
\end{abstract}

Keywords. Qubit, Quantum information transmission, Quantum teleportation, Quantum networks, Entanglement swapping, Quantum gates, CNOT gate, Wave collapse function, No cloning theorem, Reversible operations.

\section{Introduction}

One of the early quantum applications in field of quantum cryptology was demonstrated in 1992 [1], since then there has been a constant interest and research done by the scientific community in the field of quantum. Recent development of concepts and application has been seen in the areas of quantum authentication [2-4], quantum cryptology [5], quantum random number [6], distributed quantum computation [7], and quantum networks [8-10].

Quantum machines are located over different geographical locations. These machines are connected with each other over classical channels. One of the major disadvantage of such connections over classical channel is that quantum machine performs operations over qubits which has inherent parallelism and other quantum features like superposition. The qubit cannot be transferred over a classical channel and when the value of qubit is measured it collapse to a single finite value which results in loss of its quantum properties [11-15]. The inability of existing communication channels to transmit qubit has put a limit on realization of quantum computation over a network to its full potential.

One important component for any quantum network is qubit transferring/exchanging technique. In quantum mechanics operations on qubits are influenced by wave function collapse [13], No cloning theorem [14] and reversible Operations [15]. These properties pose challenges to measure or copy quantum state in between transmission and also puts restriction to use only reversible operations.

${ }^{1}$ Ankit Sharma, Department of Computer Science \& Engineering, Defense Institute of Advanced Technology, Pune, Maharashtra, India; E-mail: vividankit@yahoo.com. 
The outline of paper is as follows we first take an insight into various methods of quantum information exchange, the fundamentals of quantum mechanics. Then we cover our proposed approach, mathematical modelling, experiments and then discuss its results.

\section{Related work}

Quantum communication [16] and Quantum internet [17] has been proposed to connect various quantum devices spread over different geographical location with exchange of qubits or quantum state as the source of information.

To transmit qubit over an extended range there is a requirement of quantum repeater also there is a need of quantum router [18] for routing of information from its source to destination. Various Schemes for implementation of quantum information transmission involving quantum repeater and quantum router have been proposed. For routing function qubits are classified into control and signal qubits in which control qubits controls the path of signal qubits [18]. In some schemes function of control qubit are implemented with classical bits and classical channels which results in semi quantum devices [19]. Techniques have been demonstrated in domain of light-matter interaction [20]. Entanglement based router schemes have been proposed involving changes in signalling information as the routing process progresses [21] or not involving any changes in signalling information as the routing process progresses [22]. Routing schemes have been proposed which does not involve entanglement between control and signal qubits [23]. Other schemes for quantum information exchange have also been proposed which differ in their architectural implementation [24].

\section{Quantum preliminaries}

Before we begin to explore quantum space for quantum information exchange, we need to be familiar with few preliminaries [25].

\subsection{Qubit representation}

- In Qubit representation state 0 or $\mid 0>$ and state 1 or $\mid 1>$ is represented by a 2by-1 matrix as follow:

$$
\left|0>=\left(\begin{array}{l}
1 \\
0
\end{array}\right) \quad\right| 1>=\left(\begin{array}{l}
0 \\
1
\end{array}\right)
$$

- $\quad$ For Multi Qubits value of $|00>| 01>,, \mid 10>$ and $\mid 11>$ can be found by taking tensor product (represented by $\otimes$ ). For example, value of $\mid 00>$ can be found as:

$$
\mid 00>=\left(\begin{array}{l}
1 \\
0
\end{array}\right) \otimes\left(\begin{array}{l}
1 \\
0
\end{array}\right)=\left(\begin{array}{l}
1 \\
0 \\
0 \\
0
\end{array}\right)
$$

- Logical gates for quantum operations are also modeled with matrices. 


\subsection{Existing Techniques for quantum information transmission}

- Quantum Teleportation. It is one of the methods which can be used to transport quantum state from one place to another [26].

- Quantum entanglement swapping utilizes bell state measurement to extend the entanglement \& hence the quantum information [27-28].

\section{Proposed approach}

In this section we will delve upon our proposed approach of using CNOT gates or controlled NOT gate for quantum information transmission. We will study CNOT gate, truth table, mathematical modelling and the proposed approach.

\subsection{CNOT gates}

CNOT gate or controlled NOT gate [25] is drawn as shown in Figure 1.

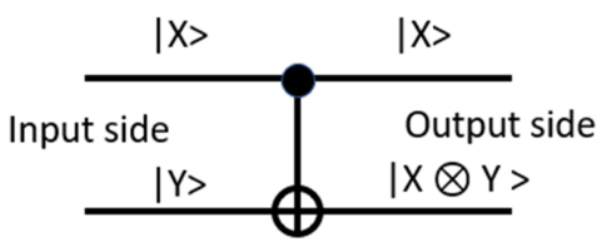

Figure 1. CNOT gate.

- This gate has two inputs and gives two outputs, the top input ( $\mid \mathrm{X}>$ ) can be called as control qubit and bottom input $(|\mathrm{Y}\rangle$ ) can be called as target qubit. If the value of control $\mid \mathrm{X}>=1$ then the value of target flips and if the value of control is $\mid X>=0$ then value of target does not change and remains same as the input. The output value of target can be depicted by the expression $|\mathrm{X}>\mathrm{XOR}| \mathrm{Y}>$ where $\mathrm{XOR}$ is the exclusive OR. It can be summarized as shown in table 1:

Table 1. Truth table CNOT gate

\begin{tabular}{|l|l|l|l|l|}
\hline \multirow{2}{*}{ S.no } & \multicolumn{2}{|c|}{ Input } & \multicolumn{2}{c|}{ Output } \\
\cline { 2 - 5 } & Control & Target & Control & Target \\
\hline $\mathbf{1}$ & $\mid 0>$ & $\mid 0>$ & $\mid 0>$ & $\mid 0>$ \\
\hline $\mathbf{2}$ & $\mid 0>$ & $\mid 1>$ & $\mid 0>$ & $\mid 1>$ \\
\hline $\mathbf{3}$ & $\mid 1>$ & $\mid 0>$ & $\mid 1>$ & $\mid 1>$ \\
\hline $\mathbf{4}$ & $\mid 1>$ & $\mid 1>$ & $\mid 1>$ & $\mid 0>$ \\
\hline
\end{tabular}

\subsection{Mathematical modelling}

- $\quad$ CNOT gate can be mathematically modelled as:

$$
\mathrm{CNOT}=\left(\begin{array}{llll}
1 & 0 & 0 & 0 \\
0 & 1 & 0 & 0 \\
0 & 0 & 0 & 1 \\
0 & 0 & 1 & 0
\end{array}\right)
$$


e.g. if we provide it with input $\mid 11>(\mid \mathrm{X}>=1$ control and $\mid \mathrm{Y}>=1$ target $)$ we get output as $\mid 10>(\mid \mathrm{X}>=1$ remains same and $\mid \mathrm{Y}>=0$ flips $)$.

- Passing $\mid 11>$ to CNOT gate can be shown as matrix multiplication as shown:

$\left(\begin{array}{llll}1 & 0 & 0 & 0 \\ 0 & 1 & 0 & 0 \\ 0 & 0 & 0 & 1 \\ 0 & 0 & 1 & 0\end{array}\right)\left(\begin{array}{l}0 \\ 0 \\ 0 \\ 1\end{array}\right)$ which gives result equal to $\left(\begin{array}{l}0 \\ 0 \\ 1 \\ 0\end{array}\right)$

Result obtained can be simplified as $\left(\begin{array}{l}0 \\ 1\end{array}\right) \otimes\left(\begin{array}{l}1 \\ 0\end{array}\right)$ which is equal to $\mid 10>$

- Similarly, other values of Table 1 can be verified by this mathematical modelling.

\subsection{Proposed methodology}

CNOT gate uses two qubits as input and gives two qubits as output if we use control qubit to pass signaling information and target qubit to pass quantum information then we can use CNOT gate for quantum information transmission.

Such an implementation would be helpful in quantum information transmission system which is working in quantum domain connected to quantum devices.

- It can be used for sharing networking information between quantum devices.

- It can be utilised for establishing quantum information transmission system.

- Control and target qubit both can be passed in the quantum network or either one of them can be passed forward depending upon the requirement and architecture of the quantum network employed.

- Such a passing of quantum information in quantum network can help build quantum networks independent of classical channels.

- Operations based on CNOT gate are faster and low cost in terms of quantum memory and processing when compared with other quantum operations like quantum teleportation.

\section{Experiment and results}

We have used IBM quantum experience an open-source platform to conduct our experiments. Figure 2 we designed a quantum circuit in which the different parameter values generated by Hadamard gate and entanglement circuit in qubits q0 and q1 are transferred/passed through different qubits q2, q3, q4, q5 signifying different values/paths.

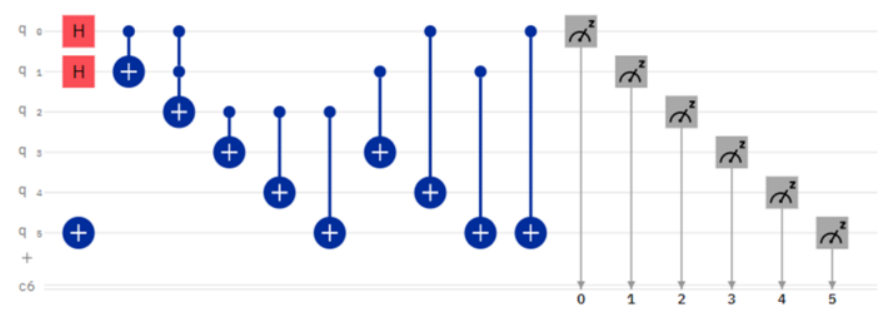

Figure 2. CNOT gate-based quantum circuit. 


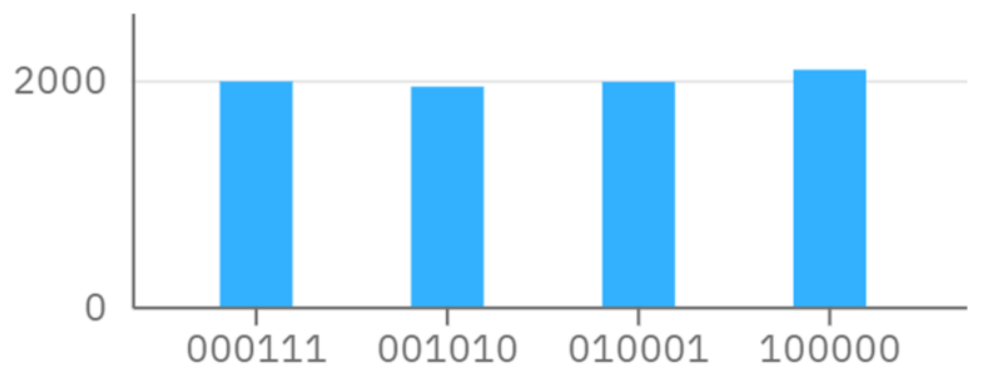

Figure 3. Result obtained after execution of circuit.

Figure 3 shows the results obtained after running the circuit on IBM quantum experience simulator. It can be observed in results that depending on values of q0 \& q1 other qubits (q2, q3, q4 \& q5) representing different values/paths are getting activated. These can be summarized as given in table 2 .

Table 2. Analysis of result obtained from execution of circuit.

\begin{tabular}{|l|l|l|l|l|}
\hline S.no & Result value & $\begin{array}{c}\text { Value of q0 } \\
\text { (Control/ Signaling } \\
\text { information) }\end{array}$ & $\begin{array}{c}\text { Value of q1 } \\
\text { (Target/ quantum } \\
\text { information) }\end{array}$ & $\begin{array}{c}\text { Path } \\
\text { activated }\end{array}$ \\
\hline $\mathbf{1}$ & 000111 & 1 & 1 & $\mathrm{q} 2$ \\
\hline $\mathbf{2}$ & 001010 & 0 & 1 & $\mathrm{q} 3$ \\
\hline $\mathbf{3}$ & 010001 & 1 & 0 & $\mathrm{q} 4$ \\
\hline $\mathbf{4}$ & 100000 & 0 & 0 & $\mathrm{q} 5$ \\
\hline
\end{tabular}

In this circuit we have chosen to activate a path on the value of control and target qubit. Similarly, we can draw circuit in which we can transfer the values of either both control and target or any one of them.

\section{Conclusion}

For transferring of qubits in quantum network, circuit based on CNOT gate can be utilized. Such a circuit would consume less resources, and would use simple quantum operations and hence would be easy to implement. Information from passing of qubits can further be enhanced with predefined quantum functions.

\section{Acknowledgement}

The authors would like to extend gratitude to the IBM quantum machines team and the many anonymous reviewers for their valuable guidance and genuine feedbacks. We would like to record our thanks to colleagues at Defense Institute of Advanced Technology, Pune for their constant motivation and technical support. 


\section{References}

[1] Bennett, C., Bessette, F., Brassard, G., Salvail, L., \& Smolin, J. (1992). Experimental quantum cryptography. Journal of Cryptology, 5(1). doi:10.1007/bf00191318.

[2] Sharma, M. K., \& Nene, M. J. (2019, October). Quantum One Time Password with Biometrics. In International Conference on Innovative Data Communication Technologies and Application (pp. 312318). Springer, Cham

[3] Sharma, MK, Nene, MJ (2020). Dual factor third-party biometric-based authentication scheme using quantum one time passwords ${ }^{\dagger}$. Security and Privacy. 2020; 3:e129. https://doi.org/10.1002/spy2.129.

[4] Upadhyay, G., \& Nene, M. J. (2016). One time pad generation using quantum superposition states. IEEE International Conference on Recent Trends in Electronics, Information \& Communication Technology.

[5] Ekert, A. K. (1991). Quantum cryptography based on Bell's theorem. Physical Review Letters, 67(6), 661-663. doi:10.1103/physrevlett.67.661

[6] M. Gupta and M. J. Nene, "Random Sequence Generation using Superconducting Qubits," 2021 Third International Conference on Intelligent Communication Technologies and Virtual Mobile Networks (ICICV), Tirunelveli, India, 2021, pp. 640-645, doi: 10.1109/ICICV50876.2021.9388495.

[7] Buhrman, H., \& Röhrig, H. (2003). Distributed Quantum Computing. Lecture Notes in Computer Science, 1-20. doi:10.1007/978-3-540-45138-9_1.

[8] Chiribella, G., D’Ariano, G. M., \& Perinotti, P. (2009). Theoretical framework for quantum networks. Physical Review A, 80(2). doi:10.1103/physreva.80.022339.

[9] Vedral, V., Barenco, A., \& Ekert, A. (1996). Quantum networks for elementary arithmetic operations. Physical Review A, 54(1), 147-153. doi:10.1103/physreva.54.147.

[10] Wallquist, M., Hammerer, K., Rabl, P., Lukin, M., \& Zoller, P. (2009). Hybrid quantum devices and quantum engineering. Physica Scripta, T137, 014001. doi:10.1088/0031-8949/2009/t137/014001.

[11] Makhlin, Y. (2002). Quantum Information Processing, 1(4), 243-252. doi:10.1023/a:1022144002391.

[12] Horodecki, R., Horodecki, P., Horodecki, M., \& Horodecki, K. (2009). Quantum entanglement. Reviews of Modern Physics, 81(2), 865-942. doi:10.1103/revmodphys.81.865.

[13] Bassi, A., Lochan, K., Satin, S., Singh, T. P., \& Ulbricht, H. (2013). Models of wave-function collapse, underlying theories, and experimental tests. Reviews of Modern Physics, 85(2), 471-527.

[14] Kuzyk, M. G. (2019). Quantum no-cloning theorem and entanglement. American Journal of Physics, $87(5), 325-327$.

[15] Brandão, F. G. S. L., \& Gour, G. (2015). Reversible Framework for Quantum Resource Theories. Physical Review Letters, 115(7). doi:10.1103/physrevlett.115.070503.

[16] P. Nema and M. J. Nene, "Pauli Matrix based Quantum Communication Protocol," 2020 IEEE International Conference on Advent Trends in Multidisciplinary Research and Innovation (ICATMRI), 2020, pp. 1-6, doi: 10.1109/ICATMRI51801.2020.9398393.

[17] Kimble, H. J. (2008). The quantum internet. Nature, 453(7198), 1023-1030. doi:10.1038/nature07127.

[18] Behera, B. K., Seth, S., Das, A., \& Panigrahi, P. K. (2019). Demonstration of entanglement purification and swapping protocol to design quantum repeater in IBM quantum computer. Quantum Information Processing, 18(4). doi:10.1007/s11128-019-2229-2

[19] Hall, M. A., Altepeter, J. B., \& Kumar, P. (2011). Ultrafast Switching of Photonic Entanglement. Physical Review Letters, 106(5). doi:10.1103/physrevlett.106.053901

[20] Aoki, T., Parkins, A. S., Alton, D. J., Regal, C. A., Dayan, B., Ostby, E., ... Kimble, H. J. (2009). Efficient Routing of Single Photons by One Atom and a Microtoroidal Cavity. Physical Review Letters, 102(8). doi:10.1103/physrevlett.102.083601.

[21] Chang, X.-Y., Wang, Y.-X., Zu, C., Liu, K., Duan, L.-M. (2012): Experimental demonstration of an entanglement-based quantum router. arXiv preprint arXiv: $1207.7265 \mathrm{v} 1$.

[22] Yan, G.-A., Cai, Q.-Y., \& Chen, A.-X. (2016). Information-holding quantum router of single photons using natural atom. The European Physical Journal D, 70(4). doi:10.1140/epjd/e2016-60724-6

[23] Lemr, K., Bartkiewicz, K., Černoch, A., Soubusta, J., \& Miranowicz, A. (2012). Experimental linearoptical implementation of a multifunctional optimal qubit cloner. Physical Review A, 85(5).

[24] Potharaju, S. P., \& Sreedevi, M. (2018). A novel cluster of quarter feature selection based on symmetrical uncertainty. Gazi University Journal of Science, 31(2), 456-470.

[25] N. S. Yanofsky and M. A. Mannucci, Quantum Computing for Computer Scientists. Cambridge, U.K.: Cambridge Univ. Press, 2008.

[26] Bouwmeester, D., Pan, J.-W., Mattle, K., Eibl, M., Weinfurter, H., \& Zeilinger, A. (1997). Experimental quantum teleportation. Nature, 390(6660), 575-579. doi:10.1038/37539.

[27] Gupta, M., \& Nene, M. J. (2020, December). Quantum Computing: An Entanglement Measurement. In 2020 IEEE International Conference on Advent Trends in Multidisciplinary Research and Innovation (ICATMRI) (pp. 1-6). IEEE. 
[28] Su, X., Tian, C., Deng, X., Li, Q., Xie, C., \& Peng, K. (2016). Quantum Entanglement Swapping between Two Multipartite Entangled States. Physical Review Letters, 117(24). 